\title{
Design of a New Multi-band Compatible Microcosmic Fractal Dipole Antenna Used in Mobile Communication
}

\author{
Bin Lin ${ }^{1}$, Danyang Qiao, a , Guopeng Kou ${ }^{1}$, Binze Chen ${ }^{1}$, Zhengzeng Wang ${ }^{1}$, \\ Qiaoli Zhu ${ }^{1}$, Xian Chen ${ }^{1}$ \\ ${ }^{1}$ Xiamen University Tan Kah Kee College, Fujian Zhangzhou 363105, China \\ aemail: qdyhawk@sina.com
}

\begin{abstract}
Keywords: Multiband compatibility; Dipole antenna; Microcosmic fractal technology; Mirroring compensation structure
\end{abstract}

\begin{abstract}
Mobile communications, which could support multi-band, multi-standard and multi-mode wireless communication technology, is one of the most important applications of wireless technology. It's the core technology for the future development of Chinese wireless communications applications. This paper focuses on the requirements of multiband compatibility in the development of mobile communications. We used plenty of advanced materials combined with the folded dipole antenna technology, microcosmic fractal technology and mirroring compensation structure to make a multi-band compatible micro-fractal dipole antenna of mobile communication. We also did a simulation analysis on performance of this antenna and produced samples in use of testing. The results show this kind of antenna has a low return loss so that it could have a larger operating bandwidth in each working band. So, the antenna successfully achieved the compatibility of a plurality of main bands in mobile communication, like GSM, TD-SCDMA, WCDMA, TD-LTE, etc.
\end{abstract}

\section{Introduction}

The Mobile communications is one of the most important applications in wireless technology [1]. On the basis of current widely used GSM mobile communications standard, China is also promoting the commercialization of TD-SCDMA. Meanwhile, WCDM as one of the 3G communication standards will occupy a position in the future Chinese market as well. So, radio signals, which are in different standards, different frequency and different formats and support mobile communications, will coexist in the space for a long time [2]. The core technology in the development of Chinese wireless communications applications is able to support Multi-band, Multi-standard and Multi-mode. At present, the GSM standard communication bands in China are 905 915MHz, $950 \sim 960 \mathrm{M} \mathrm{Hz}, 1710 \sim 1785 \mathrm{MHz}$ and $1805 \sim 1880 \mathrm{MHz}$ [3], while TD-SCDMA are $1880 \sim 1920 \mathrm{MHz}, 2010 \sim 2025 \mathrm{MHz}, 2300 \sim 2400 \mathrm{MHz}$ and WCDMA are $1920 \sim 1980 \mathrm{MHz}$, $2110 \sim 2170 \mathrm{MHz}$ [4]. However, after years of research, China's fourth-generation mobile communications technology has gradually matured and is about to enter the practical stage of commercialization. [5]. TD-LTE standard, whose independent intellectual property right entirely possessed by China, is the fourth generation of mobile communication standard and is specifically designed for mobile high-bandwidth applications. It has a broad prospect of applications. The commonly used working frequency band of TD-LTE standard is $2570 \mathrm{MHz} \sim 2620 \mathrm{MHz}$ [6].

When being used in the future, the mobile communication devices have to face such a problem that the second, third and fourth generation mobile communication systems will coexist for a long period of time. And so will different formats and standards of those generations. This requires that the future mobile communications antenna should have the function of compatibility of multi-band, while covering all operating band like GSM, TD-SCDMA, WCDMA, TD-LTE and other mobile communications standard. It will also be able to be put into a mobile communication terminal in order to meet five requirements-small size, low thickness, low return loss, high operating bandwidth and omnidirectional radiation. After comparing advantages and disadvantages of a variety of 
common antenna`s structure, we decided to use the multi-folded dipole arm antenna to achieve multi-band compatible mobile communications antenna.

\section{The introduction of dipole antenna}

Dipole antenna, which is a common antenna in the realm of wireless communication, is a kind of microstrip antenna. This dipole antenna has a simple structure and stable performance. Its size could be smaller and it could be entirely put inside a mobile communication terminal after designing and commissioning. In order to save space and decrease the size of antenna, we generally would fold dipole antenna, and try to use multi-folded dipole arm structure. This process can achieve the work of antenna in the Multi-band. The folded dipole arm antenna consists of a pair of lateral arms and the many pairs of longitudinal arms. The antenna, which respectively works in different frequency bands, overall, can be seen as a superposition of different folded dipole antennas.

\section{The introduction of mirroring compensation structure}

Mirror compensation structure is effective for improving the structure of the dipole antenna. It can also be called as a "mirror image" configuration, which means that mirror symmetrical structure has the same structure and size as metal dipole radiation patch and is installed symmetrically to metal dipole patch. Mirroring the compensation structure has many advantages like a simple structure, simple manufacturing process, low cost, and aptness of integration and so on, which can not only greatly improve the return loss of the antenna and antenna radiation performance, but also extend the operating bandwidth of the antenna. What's more, it could reduce antenna's size and surface wave and improve the efficiency of antenna and the like.

For dipole antennas, if there is a metallic conductor near them, the metal conductor would arouse current due to the electromagnetic field generated by antenna. This induced current will stimulate electromagnetic field in space, and we can call it the secondary field or the scattered field. Every point of the field within the space is the field directly inspired by the antenna and the superposition of secondary field. When we add the mirror compensation structure to the dipole antenna, you only need to adjust the distance between active dipole antenna arm and passive mirror compensation structures reasonably. Then you can make the current on mirror compensation structure and on active dipole antenna arms have the same or similar phases. At this time, any point within the space are all superimposed by the field directly inspired by antenna and the secondary field inspired by mirror the compensation structure. After that, radiation performance of the antenna will be greatly improved.

\section{The introduction of microcosmic fractal technology}

Fractal geometry is a self-similar geometric structure generated by iteration, with self-similarity between the whole and the part, as well as between the parts. Fractal design of antenna is the integration of electromagnetic theory and fractal geometry.The research found that, compared with conventional antenna, fractal antenna has a series of advantages, for instance, miniaturization, wide-band, multi-frequency operation, high radiation resistance, self-loading and so on. It can well meet the requirements of mobile communication system to the antenna. Microcosmic fractal structure is the kind of fractal structure applied to the antenna of microstrip structure. For the dipole antenna, the antenna arm has a certain width. In each $0.5 \mathrm{~mm}$ by $0.5 \mathrm{~mm}$ area of the antenna arm, the common surface fractal structure that is called Cantor is used to improve the bandwidth performance of the antenna.

From a macro perspective, antenna is the multi-folded armed dipole antenna that has certain width. Microscopically speaking, each small area of $0.5 \mathrm{~mm}$ by $0.5 \mathrm{~mm}$ is the Cantor surface fractal structure. The initial element of Cantor fractal structure is a square-shaped structure, which is divided into four rows and four columns of sixteen small squares. Remove the third small square of the first row, the first one of the second row, the fourth one of the third row and the second one of 
the fourth row, the twelve small squares remained constitute the first rank of Cantor fractal structure. Each small square of the first rank of Cantor fractal structure is divided into four rows and four columns of sixteen small squares. Remove the third smaller square of the first row, the first one of the second row, the fourth one of the third row and the second one of the fourth row; it is obtained the second rank of Cantor fractal structure. According to the iteration, it can be generated various higher ranks of Cantor fractal structure which is shown as in Figure 1 and Figure 2.

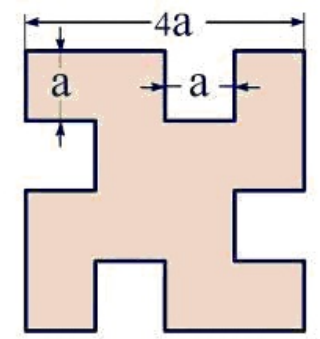

Fig.1. The scheme of the first rank of Cantor fractal structure

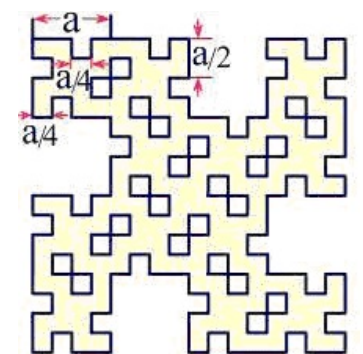

Fig.2. The scheme of the second rank of Cantor fractal structure

Microcosmic fractal structure can better balance the distribution of the RF current in the antenna with self-similarity of the fractal structure while maintaining the original frequency. Thus, it has been effectively improved the bandwidth of the antenna in each spectrum.

\section{The structural design of the new multi-band compatible microcosmic fractal dipole antenna used in mobile communication}

The typical dipole antenna is consisted of two straight wires of the same thickness and length that are arranged in a straight line. Signal is fed into the two endpoints in the middle. Thus a certain amount of current is produced and distributed on both arms of the dipole, which inspires the electromagnetic fields in the space around the antenna. When the length of a single dipole arm is $l_{0}=\lambda / 4$, the reactance component of the input impedance is zero, and then the input impedance of the antenna can be regarded as a pure resistance. Ignoring the lateral impact of the antenna's thickness, the length of the oscillator can be taken the integer times of $\lambda / 4$ in dipole antenna design.

The antenna designed in this paper is required to be compatible with second generation, the third generation and the fourth generation of mobile communication systems, while covering the GSM standard communication bands in $905 \sim 915 \mathrm{MHz}, 950 \sim 960 \mathrm{M} \mathrm{Hz}, 1710 \sim 1785 \mathrm{MHz}$ and $1805 \sim 1880 \mathrm{MHz}$, TD-SCDMA standard communication bands in $1880 \sim 1920 \mathrm{MHz}, 2010 \sim$ $2025 \mathrm{MHz}$ and $2300 \sim 2400 \mathrm{MHz}$, WCDMA standard communication bands in $1920 \sim 1980 \mathrm{MHz}$ and $2110 \sim 2170 \mathrm{MH}$, TD-LTE standard communication band in $2570 \sim 2620 \mathrm{MHz}$. 


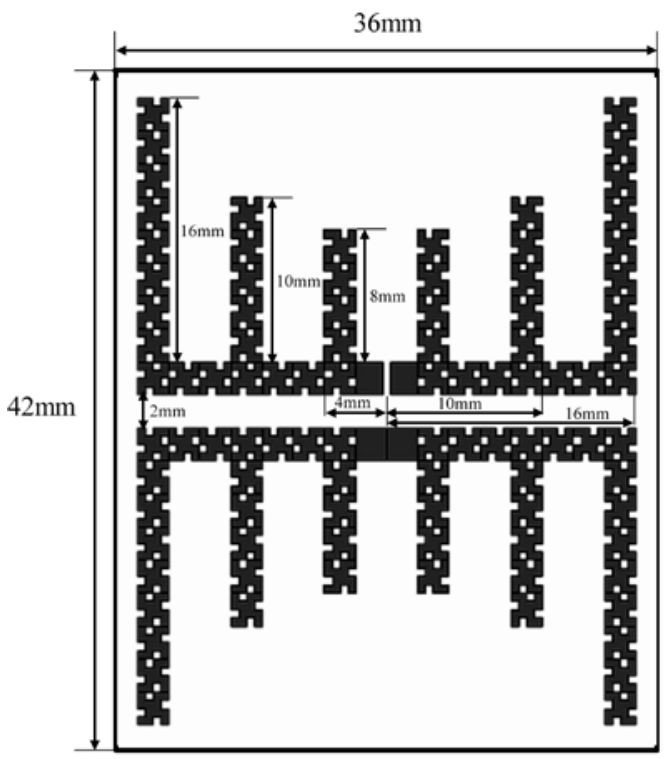

Fig.3. The scheme of the antenna structure

In the design, FR4 dielectric plate is used. The thickness of the dielectric plate is $h=2 \mathrm{~mm}$, and the relative dielectric constant is $\varepsilon_{r}=6$. The schematic structure of the antenna is shown as in Figure 3, in which the size of the antenna is $42 \mathrm{~mm}$ by $36 \mathrm{~mm}$. As shown in Figure 3, the antenna structure is consisted of two parts, of which the upper half is the active dipole antenna and the lower half is the passive mirror compensation structure. In order to reduce the size of the antenna effectively, the antenna arm has been folded. While maintaining the absolute length of the antenna arm but having it folded, the antenna size can be reduced without changing the antenna frequency. In the design, the design scheme of a half-wave dipole antenna is used. The dipole antenna arm meets the following conditions:

$$
l_{0}=\frac{c}{4 \sqrt{\varepsilon_{r}} f_{0}}
$$

Of which the free space velocity is $c=3.0 \times 10^{8} \mathrm{~m} / \mathrm{s}$. The $f_{0}$ refers to the resonant frequency of the antenna and the $l_{0}$ refers to the arm length of the antenna.

The antenna that we design is composed of one pair of lateral arms and three pairs of the longitudinal arms. The whole antenna can be regarded as the superposition of three folded dipole antennas working respectively in different frequency bands. The antenna will be divided into three working frequency bands: the first working band will cover the GSM standard communication bands in $905 \sim 915 \mathrm{MHz}$ and $950 \sim 960 \mathrm{M} \mathrm{Hz}$; the second working band will cover GSM standard communication bands in $1710 \sim 1785 \mathrm{MHz}$ and $1805 \sim 1880 \mathrm{MHz}$, TD-SCDMA standard communication bands in 1880 1920MHz and 2010 2025MHz, WCDMA standard communication bands in 1920 1980MHz and 2110 2170MH ; the third working band will cover the TD-SCDMA standard communication band in 2300 2400MHz, TD-LTE standard communication band in 2570 2620MHz. Suppose the resonant frequencies in three working bands of the antenna were $f_{01}=0.9 \mathrm{GHz}, f_{02}=1.8 \mathrm{GHz}$ and $f_{03}=2.5 \mathrm{GHz}$ respectively, corresponding antenna arms can be obtained with equation (1), which are $l_{01} \approx 34 \mathrm{~mm}, l_{02} \approx 17 \mathrm{~mm}$ and $l_{03} \approx 12 \mathrm{~mm}$ respectively. While at work, the working band of the three folded dipole antennas overlapped in one will be shifted slightly, and therefore requires subtle adjustments to be made on the design of the antenna arm length. Through simulation and debugging, we get the final design on the length of the antenna arm: $l_{01} \approx 32 \mathrm{~mm}$ (Lateral arm length is $16 \mathrm{~mm}$, and vertical arm length is $16 \mathrm{~mm}$ ), $l_{02} \approx 20 \mathrm{~mm}$ (Lateral arm length is $10 \mathrm{~mm}$, and vertical arm length is $10 \mathrm{~mm}$ ), $l_{03} \approx 12 \mathrm{~mm}$ (Lateral arm length is $4 \mathrm{~mm}$, and vertical arm length is $8 \mathrm{~mm})$.

With the adoption of the multi-folding arm structure of the dipole antenna, the radiation energy can be dispersed into multiple working bands. So the working bandwidth in each working band is 
relatively not large. To improve the bandwidth performance of the antennas in multi bands compatible with mobile communication, the Cantor micro fractal structure is used in the antenna arm. Taking into account the production

\section{The antenna's simulation analysis and experimental results}

We use the method of moments (MoM) to simulation analysis the performance of the antenna, the result of antenna's return loss performance shown in Figure 4.

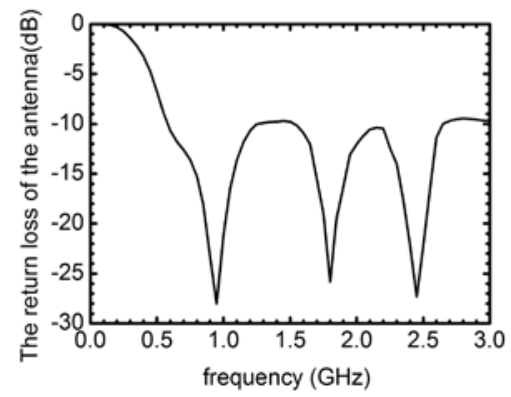

Fig.4. The simulation results of the antenna radiation characteristics

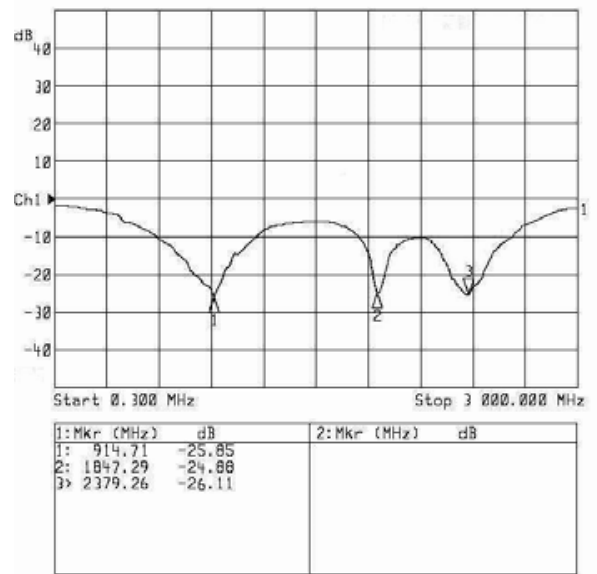

Fig.5. The experimental results of the antenna radiation characteristics

As shown in Figure 4, the three resonance center frequencies of the antenna were $0.95 \mathrm{GHz}$, $1.80 \mathrm{GHz}$ and $2.45 \mathrm{GHz}$. The return loss in value $\mathrm{S}_{11}\left(\mathrm{~S}_{11}\right.$ minimum) at the resonance frequency were $-28.03 \mathrm{~dB},-25.83 \mathrm{~dB}$ and $-27.34 \mathrm{~dB}$. When $\mathrm{S}_{11}<-10 \mathrm{~dB}$, the antenna's bandwidth within the three working bands were $0.676 \mathrm{GHz}(0.581 \sim 1.257 \mathrm{GHz}), 0.804 \mathrm{GHz}(1.384 \sim 2.188 \mathrm{GHz})$ and $0.447 \mathrm{GHz}(2.188 \sim 2.635 \mathrm{GHz})$.

After the simulation analysis, we also made an antenna samples. We adopt AV3619 Series RF integrated vector network analyzer to measure the return loss of the antenna, the results shown in Figure 5. The test results showed that the three resonance center frequency of the antenna were $0.915 \mathrm{GHz}, 1.847 \mathrm{GHz}$ and $2.379 \mathrm{GHz}$, The return loss in value $\mathrm{S}_{11}\left(\mathrm{~S}_{11}\right.$ minimum) at the resonance frequency were $-25.85 \mathrm{~dB},-24.88 \mathrm{~dB}$ and $-26.11 \mathrm{~dB}$. When $\mathrm{S}_{11}<-10 \mathrm{~dB}$, the antenna's bandwidth within the three working bands were $0.572 \mathrm{GHz}(0.583 \sim 1.155 \mathrm{GHz}), 0.401 \mathrm{GHz}(1.702 \sim 2.173$ $\mathrm{GHz})$ and $0.455 \mathrm{GHz}(2.173 \sim 2.628 \mathrm{GHz})$. The experimental results are basically consistent with the simulation results.

The simulation and experimental results show that, the antenna covers both GSM (905 915 MHz, $950 \sim 960 \mathrm{M} \mathrm{Hz}, 1710 \sim 1785 \mathrm{MHz}, 1805 \sim 1880 \mathrm{MHz})$, TD-SCDMA $(1880 \sim 1920 \mathrm{MHz}$, $2010 \sim 2025 \mathrm{MHz}, 2300 \sim 2400 \mathrm{MHz})$, WCDMA (1920 1980MHz, $2110 \sim 2170 \mathrm{MH})$, TD-LTE (2570 2620MHz) standard communications bands. It has a larger operating bandwidth in each operating band. The antenna is successfully achieved compatible with GSM, TD-SCDMA, WCDMA, TD-LTE and other mobile communication major band. 


\section{Conclusion}

This paper focuses on the requirements of multiband compatible which development of mobile communications. We use plenty of advanced materials combined with the Folded Dipole Antenna technology, Microcosmic Fractal technology and mirroring the compensation structure to make a Multi-band compatible micro-fractal dipole antenna in mobile communication. We use the method of moments (MoM) to simulation analysis the performance of the antenna and produced samples in use of testing. The simulation and experimental results show that this kind of antenna is successfully achieved compatible with GSM, TD-SCDMA, WCDMA, TD-LTE and other mobile communication major band. Currently, there are rare to combined with the Folded Dipole Antenna technology, Microcosmic Fractal technology and mirroring the compensation structure to make a mobile communication antenna-related products. Especially in the 2G, 3G, 4G mobile communications networks coexist era, it is rare to be compatible with GSM (905 $915 \mathrm{MHz}, 950 \sim$ 960M Hz, $1710 \sim 1785 \mathrm{MHz}, 1805 \sim 1880 \mathrm{MHz})$, TD-SCDMA(1880 1920MHz, $2010 \sim 2025 \mathrm{MHz}$, $2300 \sim 2400 \mathrm{MHz})$, WCDMA(1920 1980MHz, $2110 \sim 2170 \mathrm{MH})$, TD-LTE(2570 2620MHz) standard high-performance communications antenna. So this antenna has a broad application prospects.

\section{References}

[1] Ying Liu, Shuxi Gong, The antenna in the mobile communication system, Electronic Industry Press, Beijing, 2011.

[2] Lingna Liu, Jianju Liu, Development and application of 3G technology, Technology and Live. 1 (2013) 126-127.

[3] Qianhao Lv, Yong zhang, Smart antenna technology principles and progress in GSM network applications, Mobile Communications. 13 (2008) 83-85.

[4] Hui Chen, Discuss the main technical standards of 3G mobile communication, Career Horizon. 7 (2012) 174-175.

[5] Ying Chen, The fourth generation mobile communication technology, China Science and Technology Review. 33 (2013) 593.

[6] Quan Li, Analyses the TD - LTE key technologies and development trend, Chinese Electronic Commerce. 21 (2013) 75. 